\title{
PHYTOCHEMICAL, MICROBIOLOGICAL, AND TECHNOLOGICAL STUDIES IN THE FIELD OF OBTAINING A HYDROALCOHOLIC EXTRACT WITH ANTIMICROBIAL ACTIVITY FROM THE LIQUORICE ROOT
}

\author{
NIKOLAY BOYKO ${ }^{1 *}$, ALEXANDR TKACHEV ${ }^{2}$, ANATOLIY KOVALENKO ${ }^{2}$, DMITRIY PISAREV ${ }^{1}$, \\ VICTORIA KUZNIETSOVA ${ }^{3}$, NATALIA SUSHCHUK ${ }^{4}$, ALEXANDR BONDAREV ${ }^{1}$
}

\begin{abstract}
${ }^{1}$ Department of Pharmaceutical Technology, Belgorod State National Research University, Belgorod, Russia. ${ }^{2}$ Department of Veterinary Medicine, Belgorod State Agricultural University Named After V. Gorin, Federal State Budgetary Educational Institution of Higher Education, Belgorod, Russia. ${ }^{3}$ Department of Chemistry of Natural Compounds, National University of Pharmacy, Kharkiv, Ukraine. ${ }^{4}$ Department of Technology of Drugs, Odessa National Medical University, Odessa, Ukraine. Email: boykoniknik@gmail.com
\end{abstract}

Received: 27 October 2018, Revised and Accepted: 30 November 2018

\section{ABSTRACT}

Objective: The objective of this work was to carry out studies in the field of development of a hydroalcoholic extract from the liquorice root that has a high level of antimicrobial activity.

Methods: For the antibacterial study of extracts, we have used the agar well diffusion method. In our research, we have utilized six test-strain microorganisms: Escherichia coli ATCC 25922, Staphylococcus aureus ATCC 25923, Proteus vulgaris ATCC 4636, Pseudomonas aeruginosa ATCC 27853 , Candida albicans ATCC 885/653, and Bacillus subtilis ATCC 6633. Authors have applied vector theory for antimicrobial activity comparison of liquorice root extracts.

Results: Phytochemical composition and antimicrobial activity of hydroalcoholic extracts from the liquorice root with the use of different concentrations of ethanol have been studied. The optimal range of ethanol concentration of $65 \pm 15 \% \mathrm{v} / \mathrm{v}$ has been found. The dependency between the integral index of antimicrobial activity of the extract and concentration of glycyram and licuroside has been found. The minimal concentration of glycyram and licuroside in the extract has been calculated for an exhibition of the target level of extract's antimicrobial activity, which is 1.0 and $0.11 \% \mathrm{w} / \mathrm{v}$, respectively.

Conclusion: It has been found that the concentration of licuroside has greater influence on the integral index of extract's antimicrobial activity in comparison with that one of glycyram. A highly effective technology for obtaining an extract with target phytochemical parameters has been suggested.

Keywords: Liquorice root, Antimicrobial activity, Extracts, Glycyram, Licuroside, Correlation

(c) 2019 The Authors. Published by Innovare Academic Sciences Pvt Ltd. This is an open access article under the CC BY license (http://creativecommons. org/licenses/by/4. 0/) DOI: http://dx.doi.org/10.22159/ajpcr.2019.v12i1.30454

\section{INTRODUCTION}

Development and practical application of antimicrobial drugs for local wound treatment for infectious injuries of skin and mucous membrane for human/animals is a rather urgent issue [1].

It is not uncommon that for successful treatment we need drugs with several simultaneous pharmacological effects, which is impossible at present state of science and technology relating to one agent and, therefore, we need to add additional active agents with other pharmacological effects into the drug formulation.

A good alternative to mono- or combination therapy for local treatment of infectious injuries of skin and mucous membrane is to use plant extracts that may be used for development of different drug dosage forms (tablets, ointments, aerosols, solutions, sprays, etc.).

These drugs have polyfunctional properties as a result of different constituents present in their composition that has different biological effects. Many of them are accepted by the official medicine and described in National Pharmacopoeias of different countries as plant raw materials, tinctures, and extracts. However, these dosage forms still have some unsolved problems.

Despite a long-time use of tinctures and extracts in many countries, scientific grounding for choice of extractant type is still an unsolved issue in medicine and pharmacy.
The liquorice root has a long tradition of medicinal use for the treatment of different types of diseases [2,3]. Biologically active substances from the liquorice root exhibit the following effects: Antioxidant, expectorative, anti-inflammatory, anti-allergic, healing, spasmolytic, antiviral, antibacterial, and antiproliferative, etc., [4-8]. The dominant biologically active substances from Glycyrrhizae radix are glycyrrhizic acid (triterpene saponosides), chalcones (licurosid), and flavonoids (liquiritin, and liquiritigenin) [9-12]. Therefore, the development of drugs from the liquorice root with antimicrobial activity for local used is an actual task.

The aim of this work was to carry out complex studies in the field of obtaining of a hydroalcoholic extract with antimicrobial activity from liquorice root for local use both, in medical and veterinary practice.

\section{METHODS}

Plant material

For studies, we used Glycyrrhiza radices (liquorice roots) by "Krasnogorskleksredstva" company, Krasnogorsk, the Russian Federation, serial No 30417, best before $05 / 2018$. For extraction, we used plant raw material with particle size of $0.1-0.5 \mathrm{~mm}$.

Solvents and standards

As an extractant, we used water solutions of ethanol $(22,41,50,71,82$, $96 \pm 1 \% \mathrm{v} / \mathrm{v}$ ). Ethanol was of pharmaceutical grade (Russia). 
Qualitative and quantitative analyses of biologically active compounds were carried out with reference substances of licuroside and monoammonium glycyrrhizinate (glycyram) (the State Pharmacopoeia of Ukraine) and according to UV-spectra and retention times of these compounds in the high performance liquid chromatography (HPLC) profile.

\section{Preparation of extracts}

Extracts for analysis were obtained as follows: Transfer $4 \mathrm{~g}$ of the grinded plant raw material (accurately weighed) into a flask, add $20.0 \mathrm{ml}$ of the solvent (accurately weighed) and macerate for $24 \mathrm{~h}$ at $25 \pm 1^{\circ} \mathrm{C}$.

Then, the extract was decanted and centrifuged at $3000 \mathrm{rpm}$ for $30 \mathrm{~min}$; it was used for antimicrobial activity studies and analyzed by a reversed-phase HPLC.

\section{HPLC analysis}

The chromatographic studies were carried out with a chromatograph of Agilent 1200 infinity series by Agilent Technologies, the USA. The chromatographic process was carried out under the following conditions: Mobile phase (A): 1\% water solution of formic acid, second mobile phase (B): $96 \% \mathrm{v} / \mathrm{v}$ ethanol in linear gradient elution regime; chromatographic column: Supelco ascentis express $\mathrm{C}_{18} 100 \mathrm{~mm} \times 4.6 \mathrm{~mm}$ with particle size $2.7 \mu \mathrm{m}$; mobile phase velocity: $0.5 \mathrm{ml} / \mathrm{min}$; temperature of the chromatographic column: $+35^{\circ} \mathrm{C}$; and sample volume: $1 \mu \mathrm{l}[13]$.

\section{Suitability and validation parameters of the chromatographic} method

The main parameters of the validation method of analysis and suitability of HPLC system for determination of licuroside and glycyram are presented in Table 1.

\section{Method of dry residue determination}

Dry residue in the extract was determined by a gravimetric method in accordance with general pharmacopeia monograph extracts GPhA.1.4.1.0021.15 of the Russian State Pharmacopoeia [15].

\section{Antimicrobial assay}

For antimicrobial activity study, we used an agar well diffusion method. For the study, we used six test-strain microorganisms: Escherichia coli ATCC 25922, Staphylococcus aureus ATCC 25923, Proteus vulgaris ATCC 4636, Pseudomonas aeruginosa ATCC 27853, Candida albicans ATCC 885/653, and Bacillus subtilis ATCC 6633.

The microbial burden was $10^{7} \mathrm{CFU} / \mathrm{ml}$ for upper-layer of the medium. The volume of test extracts was $0.275 \pm 0.025 \mathrm{ml}$. The diameter of wells was $10 \mathrm{~mm}$, and the total width of agar layers was $4.0 \pm 0.5 \mathrm{~mm}$. Mueller-Hinton agar was used for bacteria. Sabouraud agar was used for $C$. albicans. Determination of the antimicrobial activity of extracts was carried out on two layers of solid medium in Petri dishes. $0.05 \%$ water solution of chlorhexidine gluconate and $0.5 \% \mathrm{w} / \mathrm{v}$ glycyram solution in ethanol $70 \% \mathrm{v} / \mathrm{v}$ were used as positive controls, and ethanol solutions were used as negative controls.

\section{Theory}

For ease of use and functional description of the antimicrobial activity of extracts from any physical or chemical parameters, we used vector theory, which makes it possible to describe values of extract's antimicrobial activity using several test-strains as one integral vector in $n$-dimension space. This vector is characterized by the length that describes the "strength" of extract's antimicrobial activity, $A$; with a slope angle that describes the "spectrum" of extract's action in teststrains of the microorganisms studied, $r^{2}$; with a distance between the end point of vectors (extract and a standard drug) that describes the "degree of similarity" of extract's antimicrobial properties and standard antimicrobial drug, $\Delta_{X S t}$

Calculation of the integral index of extract's antimicrobial activity, $A$, was carried out by the following formula:

$$
A=\sqrt{\left(\frac{a_{1} \cdot D_{1}}{25}\right)^{2}+\cdots+\left(\frac{a_{n} \cdot D_{n}}{25}\right)^{2}}
$$

where $A$ is an integral index of antimicrobial activity for the extract; it is a gradational and dimensionless value in case of growth inhibition zone diameter use, efficiency index range: $1.0-1.5$, the extract has weak antimicrobial activity; $1.5-2.5$, the extract has medium antimicrobial activity; $<2.5$, the extract has strong antimicrobial activity. We calculated these values by putting respective limits of growth inhibition zone diameter $(10,15$, and $25 \mathrm{~mm})$ into formula (1); $a_{1}, a_{n}$ are relative normalized weight importance coefficients of strains, species, and types of microorganisms in the diseases against which we used the extract. It is the proportion of people affected by this microorganism. The coefficient range is from 0 to 1 . In our calculations, it is taken as $1 ; D_{1} . D_{n}$ are mean values of growth inhibition zone diameters of test microorganisms, in mm. For example, $D_{1}$ is the mean value of growth inhibition zone diameter of $C$. albicans ATCC 885/653, and $D_{n}$ is the mean value of growth inhibition zone diameter of $B$. subtilis ATCC $6633 ; \delta_{D 1} . \delta_{D n}$ are errors of growth inhibition zone diameters of test microorganisms, in $\mathrm{mm} ; 25$ is normalized value, which makes it possible to transform values of growth inhibition zone diameters of test microorganisms to dimensionless values of the integral index of antimicrobial activity $(A)$ of the extracts.

The correlation coefficient $(\cos \gamma=r$ ) between vectors and distance between the end point of vectors $\left(\Delta_{\mathrm{XS}}\right)$ was calculated by the following formulas:

$$
\cos \gamma=r=\frac{\Sigma\left[a_{i}^{X} \cdot D_{i}^{X} \cdot a_{i}^{S t} \cdot D_{i}^{S t}\right]}{\sqrt{\Sigma\left[a_{i}^{X} \cdot D_{i}^{X}\right]^{2}} \cdot \sqrt{\Sigma\left[a_{i}^{S t} \cdot D_{i}^{S t}\right]^{2}}} a
$$

Table 1: Main parameters of the validation method of analysis and suitability of HPLC system for determination of licuroside and

\begin{tabular}{|c|c|c|c|}
\hline \multirow[t]{2}{*}{ Parameter } & \multirow[t]{2}{*}{ Pharmacopeia limitation [14] } & \multicolumn{2}{|l|}{ Compound } \\
\hline & & Licuroside & Glycyram \\
\hline Retention time $\left(\mathrm{t}_{\mathrm{R}}\right)$, min & - & $21.7 \pm 0.3$ & $35.3 \pm 0.3$ and $35.9 \pm 0.3$ \\
\hline Asymmetry coefficient (T) & $\leq 2.0$ & 0.8 & 0.68 and 0.72 \\
\hline Separation coefficient $\left(\mathrm{R}_{\mathrm{s}}\right)$ & $\geq 1.5$ & 2.31 & 1.65 and 1.50 \\
\hline Theoretical plates number $(\mathrm{N})$ & $\geq 1000$ & 169,803 & 699,649 and 888,042 \\
\hline RSD of peak's area, \% & $\leq 2.0$ & 1.7 & 1.9 and 1.9 \\
\hline $\mathrm{LOD}, \mathrm{g} / \mathrm{ml}$ & - & $2.0 \cdot 10^{-5}$ & $8.9 \cdot 10^{-5}$ \\
\hline $\mathrm{LOQ}, \mathrm{g} / \mathrm{ml}$ & - & $6.1 \cdot 10^{-5}$ & $2.7 \cdot 10^{-4}$ \\
\hline Determination coefficient, $r^{2}$ & $\geq 0.99$ & 0.9999 & 0.9997 \\
\hline Calibration linear equation, $C(\mathrm{~g} / \mathrm{ml})=f(S(m A U \cdot s)$ & - & $C=(2.29 \pm 0.04) \cdot 10^{-7} \cdot S$ & $C=(1.77 \pm 0.06) \cdot 10^{-6} \cdot S$ \\
\hline
\end{tabular}
glycyram

HPLC: High performance liquid chromatography 


$$
\Delta_{X S t}=\sqrt{\left(\frac{a_{1} \cdot\left(D_{1}-D_{1}^{S t}\right)}{25}\right)^{2}+\cdots+\left(\frac{a_{n} \cdot\left(D_{n}-D_{n}^{S t}\right)}{25}\right)^{2}}
$$

where $a_{i}^{X}, \ldots a_{i}^{S t}$ are relative normalized weight importance coefficients of test-strains for the test extract - X and "standard" - St. In our calculations, it is taken as $1 ; D_{i}^{X} \ldots D_{i}^{S t}$ are mean values of growth inhibition zone diameters of test microorganisms, for the test extract - $X$ and for "standard" - St, in mm.

In our research, we used a virtual vector as "standard" with some conventional coordinates for all test-strain, for example, $D_{i}^{S t}=25.0 \mathrm{~mm}$ (for growth inhibition zone diameters of test microorganisms). This value was chosen because it is a breakpoint between such categories of microorganism sensitivity to antimicrobials as intermediate and sensitive.

\section{Regression analysis}

For the construction of the regression model of dependency of the integral index of extract's antimicrobial activity from the glycyram and licuroside concentration, we used table curve 3D v4.0 program.

\section{RESULTS AND DISCUSSION}

In the first series of experiments, phytochemical studies of extracts by HPLC and gravimetric methods have been carried out. The results of the quantitative analysis of licuroside, glycyram, and dry residue are presented in Table 2 .
As it can be seen from the data of Table 2, maximum concentration of licuroside is observed in the extract based on ethanol $71 \% \mathrm{v} / \mathrm{v}$, and for glycyram, in the extract based on ethanol $41 \% \mathrm{v} / \mathrm{v}$, moreover, in the extract based on ethanol $96 \% \mathrm{v} / \mathrm{v}$, its concentration was 173 -fold less than in the extract based on ethanol $41 \% \mathrm{v} / \mathrm{v}$. These data agree with our previous studies [13].

In the second series of experiments, studies of the antimicrobial activity of extracts by in vitro agar diffusion methods, the variant of "wells," has been carried out. The results of the antimicrobial activity of extracts and reference drugs are presented in Table 3.

As it can be seen from Table 3, all extracts demonstrate the high level of antimicrobial activity, but its maximum value was detected for the extract with ethanol $71 \% \mathrm{v} / \mathrm{v}$. Antimicrobial activity of this extract is almost similar to $0.05 \%$ chlorhexidine gluconate solution.

For the possibility of comparison of the integral parameter of drugs' antimicrobial activity between each other, we used vector algebra theory. In this connection, the results related to calculations of some important parameters using formulas (1)-(3) are presented in Table 4.

As it can be seen from Table 4, the integral index of antimicrobial activity of the extract based on ethanol $71 \% \mathrm{v} / \mathrm{v} \mathrm{A}=2.08$ has the equal value to the reference drug chlorhexidine gluconate $A=2.07$, but its vector is closely adjacent to the virtual drug in terms of parameter $\Delta_{A S t}=0.44$ then the vector of the reference drug $\Delta_{A S t}=0.51$. This can be explained by the fact that extract's antimicrobial activity has less variation among the

Table 2: Concentration of licuroside, glycyram, and dry residue in the extracts obtained from ethanol-water solutions with different concentrations

\begin{tabular}{|c|c|c|c|c|c|c|c|}
\hline \multirow[t]{3}{*}{ Compound $(\lambda, \mathbf{n m})$} & \multirow[t]{3}{*}{ Retention time, min* } & \multicolumn{6}{|c|}{ Compound concentration, $\% \mathrm{w} / \mathrm{v}$} \\
\hline & & \multicolumn{6}{|c|}{ Ethanol, \% v/v } \\
\hline & & 22 & 41 & 50 & 71 & 82 & 96 \\
\hline $\begin{array}{l}\text { 1. Licuroside concentration, } \\
\% \mathrm{w} / \mathrm{v}(360.4 \mathrm{~nm})\end{array}$ & $21.7 \pm 0.3$ & $0.098 \pm 0.003$ & $0.108 \pm 0.003$ & $0.133 \pm 0.004$ & $0.170 \pm 0.005$ & $0.149 \pm 0.005$ & $0.067 \pm 0.002$ \\
\hline $\begin{array}{l}\text { 2. Glycyram concentration, } \\
\% \mathrm{w} / \mathrm{v}(258.4 \mathrm{~nm})\end{array}$ & $35.3 \pm 0.3$ and $35.9 \pm 0.3$ & $1.46 \pm 0.04$ & $1.53 \pm 0.05$ & $1.55 \pm 0.08$ & $1.45 \pm 0.04$ & $1.02 \pm 0.03$ & $0.0088 \pm 0.0003$ \\
\hline 3. Dry residue, \% w/v & - & $6.6 \pm 0.2$ & $6.4 \pm 0.2$ & $6.2 \pm 0.2$ & $6.1 \pm 0.2$ & $4.0 \pm 0.2$ & $1.62 \pm 0.08$ \\
\hline
\end{tabular}

Table 3: Antimicrobial activity of extracts from liquorice roots

\begin{tabular}{|c|c|c|c|c|c|c|c|}
\hline \multirow[t]{3}{*}{ No. } & \multirow[t]{3}{*}{ Test-strain microorganism } & \multicolumn{6}{|c|}{ Growth inhibition zone diameters of test microorganisms, in $\mathrm{mm}^{*}$} \\
\hline & & \multicolumn{4}{|c|}{ Ethanol concentration, $\% \mathrm{v} / \mathrm{v}$} & \multirow{2}{*}{$\frac{\mathrm{Ch}^{* *}}{\mathrm{Gl}}$} & \multirow[t]{2}{*}{$\mathrm{NC}^{* * *}$} \\
\hline & & 22 & 41 & 71 & 96 & & \\
\hline 1. & Staphylococcus aureus ATCC 25923 & $20.3 \pm 1.4$ & $20.0 \pm 1.4$ & $24.3 \pm 1.4$ & $20.7 \pm 1.4$ & $\frac{24.3 \pm 1.4}{16.0 \pm 1.4}$ & Growth \\
\hline 2. & Escherichia coli ATCC 25922 & $21.0 \pm 1.4$ & $20.3 \pm 1.4$ & $22.3 \pm 1.4$ & $18.7 \pm 1.4$ & $\frac{20.7 \pm 1.4}{14.7 \pm 1.4}$ & Growth \\
\hline 3. & Pseudomonas aeruginosa ATCC 27853 & $18.3 \pm 1.4$ & $19.0 \pm 1.4$ & $20.3 \pm 1.4$ & $20.3 \pm 1.4$ & $\frac{17.0 \pm 1.4}{14.0 \pm 1.4}$ & Growth \\
\hline 4. & Proteus vulgaris ATCC 4636 & $16.7 \pm 1.4$ & $17.7 \pm 1.4$ & $19.7 \pm 1.4$ & $18.0 \pm 1.4$ & $\frac{16.3 \pm 1.4}{13.7 \pm 1.4}$ & Growth \\
\hline 5. & Bacillus subtilis ATCC 6633 & $22.7 \pm 1.4$ & $23.3 \pm 1.4$ & $22.7 \pm 1.4$ & $22.3 \pm 1.4$ & $\frac{22.7 \pm 1.4}{16.7 \pm 1.4}$ & Growth \\
\hline 6. & Candida albicans ATCC 885/653 & $14.7 \pm 1.4$ & $16.7 \pm 1.4$ & $19.3 \pm 1.4$ & $19.3 \pm 1.4$ & $\frac{24.3 \pm 1.4}{13.3 \pm 1.4}$ & Growth \\
\hline
\end{tabular}

${ }^{*}$ Repeat counts $\mathrm{n}=3$, significance level $\mathrm{p}=0.95 ;{ }^{*} \mathrm{Ch}$ is $0.05 \% \mathrm{w} / \mathrm{v}$ chlorhexidine gluconate in water solution and $\mathrm{Gl}$ is $0.5 \% \mathrm{w} / \mathrm{v}$ glycyram solution in ethanol $71 \% \mathrm{v} / \mathrm{v}$

${ }^{* * *} \mathrm{C}$ is negative control are ethanol solutions from 22 to $96 \% \mathrm{v} / \mathrm{v}$ 
test strains of microorganisms than the reference drug. From the data in Table 4, we can also see that the square correlation coefficient $\left(r^{2}\right)$ for all tested extracts and reference drugs have a high value of $r^{2}=0.99$, this value demonstrates that extracts and drugs inhibit the growth of all test strains of microorganisms.

At the next stage of our study, we carried out analysis of data and chose the type of correlation model for dependency between the integral index of extract's antimicrobial activity and concentration of glycyram and licuroside. This stage was carried out with the use of table curve 3D v4.0 program.

From mathematical equations proposed by the program, we chose only one equation that in our opinion was the most adequate for an explanation of the dependency of the integral index of extract's antimicrobial activity and concentration of dominant components in the extract (glycyram and licuroside).

The equation found is presented below:

$$
\mathrm{A}=\mathrm{a}+b \ln C_{G}+d \ln C_{L}
$$

Where $A$ is an integral index of extract's antimicrobial activity; $a$ is a constant that equals $4.22 \pm 0.2 ; b$ is a constant that equals - (minus) $0.041 \pm 0.004 ; C_{G}$ is glycyram concentration, $\mathrm{g} / \mathrm{ml} ; d$ is a constant that equals $0.36 \pm 0.03$; $C_{L}$ is licuroside concentration, $\mathrm{g} / \mathrm{ml}$.

Determination coefficient of this equation is $r^{2}=0.99$; moreover, all constants in the equation are significant and have the confidence interval of variation, not more than $10 \%$
Analysis of Equation (4) demonstrates that the coefficient at logarithm of licuroside concentration is greater than the coefficient at logarithm of glycyram concentration $(0.36>0.041)$, which indicates to the dominant influence of licuroside concentration in the extract on the integral index of antimicrobial activity.

Moreover, the minus sign at the coefficient related to logarithm of glycyram concentration indicates to the fact that higher concentration of this substance in the extract leads to decrease of the integral index of antimicrobial activity that is an unexpected fact because as it can be seen from the data of Tables 3 and 4, this compound possesses antimicrobial activity.

Then, using the regression equation obtained and addition data on phytochemical composition of extracts obtained with other ethanol concentrations (Table 2), we found the minimal values of glycyram and licuroside concentration, $1.0 \% \mathrm{w} / \mathrm{v}$ and $0.11 \% \mathrm{w} / \mathrm{v}$, respectively, for exhibition of the integral index of extracts of antimicrobial activity with a target level of $A=2.00 \pm 0.06$

Thus, for development of production technology for obtaining a hydroalcoholic extract from the liquorice root with maximum antimicrobial activity level, we should use ethanol with concentration $65 \pm 15 \% \mathrm{v} / \mathrm{v}$. Therein, for standardization of the extract, we should take into account concentrations of both licuroside and glycyram.

At the final stage of our work, we carried out technological studies on obtaining of the extract with target concentration values of glycyram and licuroside by the filtration method of extraction.

Table 4: Integral antimicrobial parameters of extracts from liquorice roots

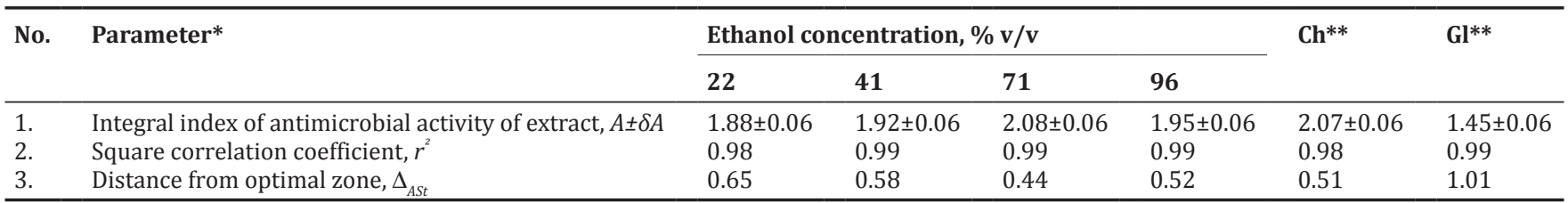

${ }^{*}$ Statistical calculations are carried out with repeat counts $\mathrm{n}=3$ and significance level $\mathrm{p}=0.95 ;{ }^{* *} \mathrm{Ch}$ is $0.05 \% \mathrm{w} / \mathrm{v}$ chlorhexidine gluconate in water solution and $\mathrm{Gl}$ is $0.5 \%$ $\mathrm{w} / \mathrm{v}$ glycyram solution in ethanol $71 \% \mathrm{v} / \mathrm{v}$

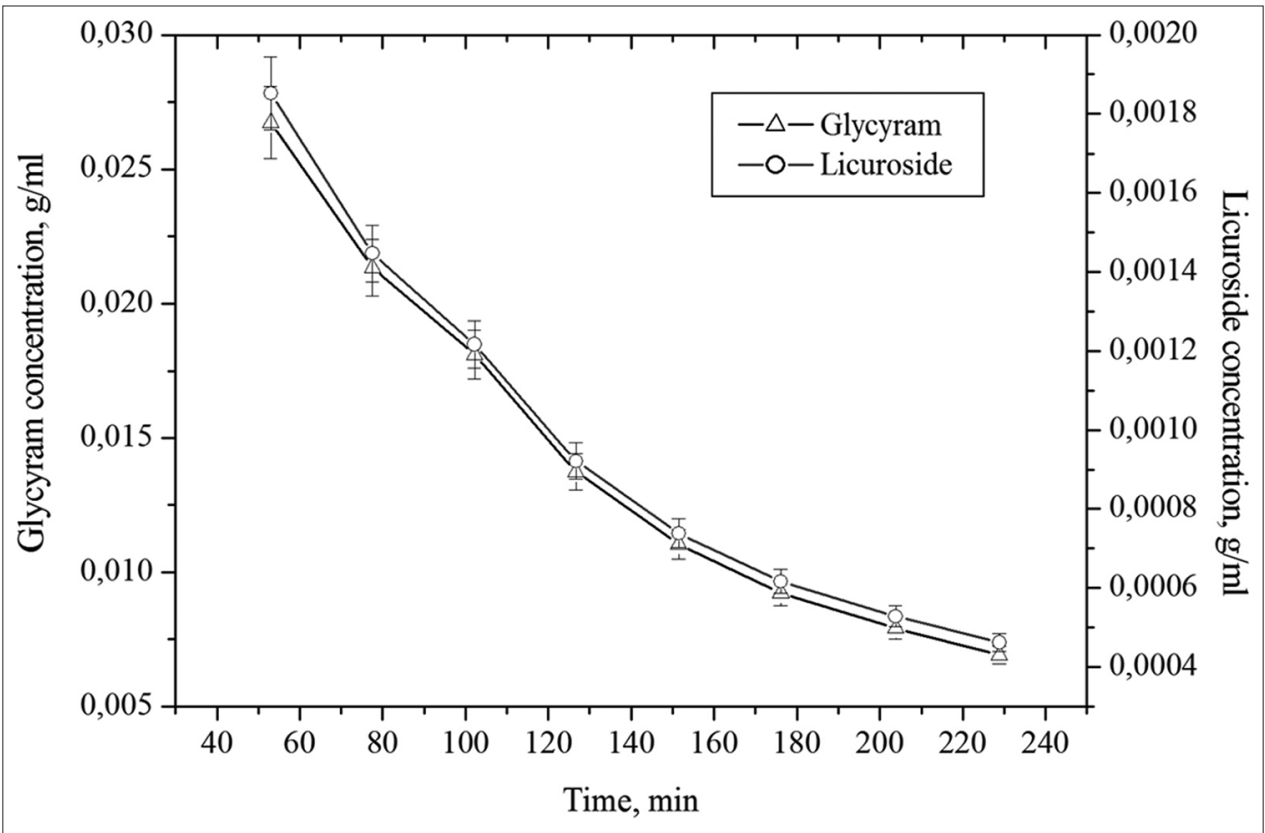

Fig. 1: Diagram of dependency between the concentration of biologically active substances and the time 
Fig. 1 presents a diagram of dependency between the concentration of biologically active substances and the time of extract drainage at 1:1 ratio of volume extract/weight of plant raw material.

As it can be seen from the data in Fig. 1, critical concentrations of glycyram $(0.018 \mathrm{~g} / \mathrm{ml})$ and licuroside $(0.0012 \mathrm{~g} / \mathrm{ml})$ in the extract are observed at the third drainage volume. Thus, from the technological point of view, it is more reasonable to obtain the extract with the composition determined at $25^{\circ} \mathrm{C}$, plant raw material/extract ratio $1: 3$ (w/v), and extraction time $100 \mathrm{~min}$.

In accordance with the mathematical model, the integral index of extract's antimicrobial activity for these concentrations of licurosid and glycyram should be equal to $A=1.97$, which is within the suggested range.

These results form the background for the development of a rational technology for obtaining the extract from the liquorice root with maximum antimicrobial activity, as well as for analytical control of its quality.

\section{CONCLUSION}

Phytochemical and microbiological analyses of extracts from the liquorice root obtained with the use of different concentrations of ethanol have been carried out. Regression analysis of experimental data has been performed. An empirical dependency between the integral index of extract's antimicrobial activity and glycyram and licuroside concentrations has been found. Minimal licuroside and glycyram concentrations in the extract for an exhibition of its target level of antimicrobial activity have been calculated. Using a filtration method of extraction, we obtained the extract that satisfies minimal phytochemical requirements related to glycyram and licuroside content.

\section{ACKNOWLEDGMENT}

Authors express their highest esteem and thanks to Doct. Chem. Sc., Prof. Vasiliy Ivanovich Litvinenko, the Head of the Laboratory of Chemistry and Technology of Phytochemical Drugs of State Enterprise "State Scientific Center of Drugs and Medicinal Products," Kharkiv, Ukraine, for his help with reference substances.

\section{AUTHORS' CONTRIBUTIONS}

All authors had equally contributed to the research work.

\section{CONFLICTS OF INTEREST}

The authors declare that they have no conflicts of interest.

\section{REFERENCES}

1. McCulloch JM, Kloth LC. Wound Healing: Evidence-Based Management. $4^{\text {th }}$ ed. Philadelphia, PA: F.A. Davis Company; 2010.

2. Asl MN, Hosseinzadeh H. Review of pharmacological effects of Glycyrrhiza sp. And its bioactive compounds. Phytother Res 2008:22:709-24.

3. Baltina LA, Kondratenko RM, Baltina LM Jr., Plyasunova OA, Pokrovskii AG, Tolstikov GA. Prospects for creation of new antiviral drugs based on glycyrrhizic acid and its derivatives a review. Pharm Chem J 2009;43:539-48.

4. Delbò M. Assessment Report on Glycyrrhiza glabra L. and or Glycyrrhiza inflate Bat. and or Glycyrrhiza uralensis Fisch., Radix. Report No. EMA/HMPC/571122/2010. London: Committee on Herbal Medicinal Products (HMPC), European Medicines Agency; 2013.

5. Lakshmi T, Geetha R. Glycyrrhiza glabra Linn. Commonly known as Liquorice: A therapeutic review. Int J Pharm Pharm Sci 2011;3:20-5.

6. Langer D, Czarczynska-Gpslinska B, Goslinski T. Glycyrrhetinic acid and its derivatives in infectious diseases. Curr Issues Pharm Med Sci 2016;29:118-23.

7. Shinde D, Koratkar S, Sharma N, Shitole A. Antioxidant activity and antiproliferative action of methanolic extract of liquorice (Glycyrrhiza glabra) in HEPG2 cell line. Int J Pharm Pharm Sci 2016;8:293-8.

8. Gaitry CP, Binda DS, Farhin I, Sujata SD. Antimicrobial and antioxidant activities of methanol extract roots of Glycyrrhiza glabra and HPLC analysis. Int J Pharm Pharm Sci 2013;5:975-1491.

9. Litvinenko VI, Georgievskiy VP, Fursa NS, Ammosov AS, Popova TP. Liquorice: Taxonomy, Chemistry, Technology, Standardization, Pharmacology, Clinic. Yaroslavl: Avers Plus; 2014

10. Popova NV, Litvinenko VI, Kucanjan AS. Medicinal Plants of the World: Encyclopedic Handbook. Kharkov: Disa Plus; 2016.

11. Tolstikov GA, Baltina LA, Grankina VP, Kondratenko RM, Tolstikova TG. Liquorice: Biodiversity, Chemistry, Application in Medicine. Novosibirsk: Academic Publishing House "Geo"; 2007.

12. World Health Organization. Monographs on Selected Medicinal Plants. Vol. 1. Geneva: World Health Organization; 1999.

13. Boyko NN, Pisarev D, Zhilyakova E, Novikov O, Kuznietsova V, Sushchuk N, et al. Modeling of solvent effects on phytocompounds' extraction from Glycyrrhizae radix. J Adv Pharm Technol Res 2018;9:66-72

14. European Pharmacopoeia Commission. European Pharmacopoeia. $8^{\text {th }}$ ed. Strasbourg: European Directorate for the Quality of Medicines and HealthCare; 2014.

15. Russian Healthcare Ministry. Russian State Pharmacopoeia. $13^{\text {th }}$ ed. Vol. 2. Moscow: Russian Healthcare Ministry; 2015. 